\title{
BAND OFFSETS AT STRAINED-LAYER INTERFACES
}

\section{CHRIS G. VAN DE WALLE}

IBM Thomas J. Watson Research Center, P.O Box 218, Yorktown Heights, NY 10598

\begin{abstract}
Strained-layer heterojunctions and superlattices have recently shown tremendous potential for device applications because of their flexibility for tailoring the electronic band structure. We present a theoretical model to predict the band offsets at both latticematched and pseudomorphic strained-layer interfaces. The theory is based on the localdensity-functional pseudopotential formalism, and the "model solid approach" of Van de Walle and Martin. The results can be most simply expressed in terms of an "absolute" energy level for each semiconductor, and deformation potentials that describe the effects of strain on the electronic bands. The model predicts reliable values for the experimentally observed lineups in $\mathrm{Si} / \mathrm{Ge}, \mathrm{GaAs} / \mathrm{InAs}$, and $\mathrm{ZnSe} / \mathrm{ZnS}$ systems, and can be used to explore which combinations of materials and configurations of the strains will lead to the desired electronic properties.
\end{abstract}

\section{INTRODUCTION}

In recent years, tremendous developments have occurred in the field of semiconductor heterojunctions and superlattices and their applications in electronic devices. The introduction and improvement of novel growth techniques (in particular molecular beam epitaxy) have made it possible to produce extremely high-quality epitaxial interfaces, even between materials which differ in lattice constant by several percent. This lattice mismatch can be accommodated by uniform lattice strain in sufficiently thin layers. Such a "pseudomorphic" interface is characterized by an in-plane lattice constant which remains the same throughout the structure. The strains can cause profound changes in the electronic properties, and therefore provide extra flexibility in device design. Knowledge of the discontinuities in valence and conduction bands at semiconductor interfaces is essential for the analysis of the properties of any heterojunction, but has remained rather limited due to experimental difficulties, and the absence of reliable theoretical predictions.

Only recently has it become possible to perform first-principles calculations of the band offsets at a semiconductor interface. Such calculations, based on local-densityfunctional theory and ab initio pseudopotentials, have been carried out for a wide variety of lattice-matched interfaces, ${ }^{1}$ and also for representative examples of strained-layer interfaces. $^{2}$ Unfortunately, the computational complexity of such calculations is very high, which limits their use as a tool in the exploration and design of novel heterostructures. Particularly in the case of strained-layer interfaces, carrying out a self-consistent calculation for every imaginable strain configuration would be unfeasible. This clearly illustrates the need for a reliable model theory that can predict band offsets for a wide variety of interfaces without the need for heavy calculations. Several model theories have been developed in the past, with variable degrees of success. ${ }^{3}$ The so-called "model solid" theory that we will discuss here yields results for lattice-matched interfaces which are at least as good as those achieved by other model theories. Even more importantly, it provides a 
natural way of dealing with strained-layer interfaces. None of the other model theories includes a prescription for incorporating strain; attempts to add these effects a posteriori have not been very successful so far. In this paper, we will therefore concentrate on the features of the model-solid theory that allow us to examine strained layers.

\section{THE MODEL SOLID THEORY}

The model theory, and its connection to the full first-principles calculations, has been described in detail elsewhere. ${ }^{1,4,5}$ Here we will only summarize the underlying theory for the purposes of the present applications. First, we point out that throughout this paper we assume all interfaces to be ideal, i.e. the bulk atomic structure of each of the semiconductors is maintained up to the interface. For strained-layer interfaces, we include the appropriate strains in each of the materials to construct a pseudomorphic interface.

Our theory relies on two main components: first, the generation of an accurate band structure, and second, the alignment of this band structure on an "absolute" energy scale. The first part is accomplished by performing density-functional calculations on individual bulk semiconductors, described by ab initio pseudopotentials. ${ }^{6}$ It is well established by now that such calculations produce reasonable band structures, and that changes in the bands induced by hydrostatic or uniaxial strains are reliably predicted. A discussion of intrinsic deficiencies of density-functional theory and their effect on band lineups was presented in Refs. 1 and 2.

The second part of the problem is that of establishing an absolute energy scale. Such an absolute reference can only be present when the energies in the bulk semiconductor can be referred to the "vacuum level". Since typical bulk calculations are carried out for an infinite crystal, no such reference is available; the calculated energy bands are referred to an average electrostatic potential within the solid, which is only defined to within an arbitrary constant. Our model consists of a particular way of relating this average electrostatic potential to the vacuum level. This puts all calculated energies on an absolute energy scale, and allows us to derive band lineups by simply subtracting values for individual semiconductors. The common reference is accomplished by modeling the solid as a superposition of neutral atoms. In each atom, the electrostatic potential is rigorously defined with respect to the vacuum level. The average electrostatic potential in this "model solid" is therefore, by superposition, also well specified on the absolute energy scale. We should emphasize that this choice of an absolute energy scale is certainly not unique. It is, however, well defined by our prescription of superposition of neutral atomic ${ }^{7}$ charge densities, calculated within the LDA for the pseudopotentials that we use in the band-structure calculations.

Table I contains an overview of our results for elemental, III-V and II-VI semiconductors. Some of the values for lattice constants listed in Table $I$ are slightly different from the exact experimental values. That is because, in an attempt to establish classes of closely lattice-matched materials, we decided to neglect any mismatch that is less than $\sim 0.5 \%$. Such a small mismatch would only lead to strain effects in the lineups which are significantly smaller than the accuracy of the present calculations (and of most experimental measurements). Listed in the Table are values for $\mathrm{E}_{\mathrm{v}, \mathrm{av}}$, which is the weighted average over the three uppermost valence bands at $\Gamma$ (known as the light and heavy hole bands, and the spin-orbit split-off band).

Besides the position of the valence band on an absolute energy scale, $E_{v, a v}$, the model-solid approach can also give us information about the variation of this energy when 


\section{TABLE I}

Lattice constant a, spin-orbit splitting $\Delta_{0}$, and gap $E_{\mathrm{g}}$ of various semiconductors. ${ }^{10}$ Values of $E_{v, a v}$ (the weighted average over the three uppermost valence bands at $\Gamma$ ), $\mathrm{a}_{\mathrm{v}}=\mathrm{d}\left(\mathrm{E}_{\mathrm{v}, \mathrm{av}}\right) / \mathrm{d}(\ln \Omega), \quad \mathrm{a}_{\mathrm{c}}=\mathrm{d}\left(\mathrm{E}_{\mathrm{c}}\right) / \mathrm{d}(\ln \Omega)$, and $\mathrm{a}=\mathrm{d}\left(\mathrm{E}_{\mathrm{c}}-\mathrm{E}_{\mathrm{v}, \mathrm{av}}\right) / \mathrm{d}(\ln \Omega)$ are calculated within the model-solid approach. For indirect-gap semiconductors, values of $E_{g}, a_{c}$, and a are given for both direct and indirect gaps. Lattice constants are in $\AA$, all other values are in $\mathrm{eV}$.

\begin{tabular}{|c|c|c|c|c|c|c|c|c|c|c|}
\hline & $\mathrm{a}(\AA)$ & $\Delta_{0}$ & $E_{B}^{d r}$ & $\mathrm{E}_{g}^{\mathrm{ind}}$ & $E_{v, a v}$ & $a_{v}$ & $a_{c}^{d i r}$ & $a^{\text {dir }}$ & $a_{c}^{\text {and }}$ & $a^{\text {nnd }}$ \\
\hline $\mathrm{Si}$ & 5.43 & 0.04 & 3.37 & 1.17 & -7.03 & 2.46 & 1.98 & -0.48 & 4.18 & 1.72 \\
\hline $\mathrm{Ge}$ & 5.65 & 0.30 & 0.89 & 0.74 & -6.35 & 1.24 & -8.24 & -9.48 & -1.54 & -2.78 \\
\hline GaAs & 5.65 & 0.34 & 1.52 & & -6.92 & 1.16 & -7.17 & -8.33 & & \\
\hline AlAs & 5.65 & 0.28 & 3.13 & 2.23 & -7.49 & 2.47 & -5.64 & -8.11 & 4.09 & 1.62 \\
\hline $\operatorname{In} A s$ & 6.08 & 0.38 & 0.41 & & -6.67 & 1.00 & -5.08 & -6.08 & & \\
\hline $\mathrm{GaP}$ & 5.43 & 0.08 & 2.90 & 2.35 & -7.40 & 1.70 & -7.14 & -8.83 & 3.26 & 1.56 \\
\hline AlP & 5.43 & - & 3.63 & 2.51 & -8.09 & 3.15 & -5.54 & -8.70 & 5.12 & 1.97 \\
\hline $\operatorname{InP}$ & 5.87 & 0.11 & 1.42 & & -7.04 & 1.27 & -5.04 & -6.31 & & \\
\hline $\mathrm{GaSb}$ & 6.08 & 0.82 & 0.75 & & -6.25 & 0.79 & -6.85 & -7.64 & & \\
\hline $\mathrm{AlSb}$ & 6.08 & 0.65 & 2.32 & 1.70 & -6.66 & 1.38 & -6.97 & -8.36 & 3.05 & 1.67 \\
\hline $\mathrm{InSb}$ & 6.48 & 0.81 & 0.24 & & -6.09 & 0.36 & -6.17 & -6.53 & & \\
\hline $\mathrm{ZnSe}$ & 5.65 & 0.43 & 2.83 & & -8.37 & 1.65 & -4.17 & -5.82 & & \\
\hline $\mathrm{ZnS}$ & 5.40 & 0.07 & 3.84 & & -9.15 & 2.31 & -4.09 & -6.40 & & \\
\hline $\mathrm{CdTe}$ & 6.48 & 0.93 & 1.59 & & -7.07 & 0.55 & -3.96 & -4.52 & & \\
\hline $\mathrm{HgTe}$ & 6.48 & 1.05 & -.30 & & -6.88 & -.13 & -4.60 & -4.48 & & \\
\hline
\end{tabular}


strain is present in the system. Uniaxial components of the strain can have a profound effect on degenerate bands; they lead to splittings of the valence bands which we will discuss later. These splittings are averaged out, however, when considering the average $\mathrm{E}_{\mathrm{y}, \mathrm{uv}}$, which will be subject only to shifts due to the hydrostatic component of the strain (corresponding to a volume change). Once again, two contributions occur in the calculation. On the one hand, there is the effect on the band structure when the solid is compressed; the bands will shift with respect to the average potential in the solid. On the other hand, the average potential itself will be shifted due to the (hydrostatic component of the) strain, through the volume-averaging of the potential. The total effect leads to a hydrostatic deformation potential for the valence band:

$$
\mathrm{a}_{\mathrm{v}}=\mathrm{d}\left(\mathrm{E}_{\mathrm{v}, \mathrm{av}}\right) / \mathrm{d}(\ln \Omega)
$$

which expresses the shift (in eV) in $E_{\mathrm{v}, \mathrm{av}}$ per unit fractional volume change. A similar definition applies to the conduction band deformation potential $a_{c}$. The band-gap deformation potential is of course equal to $a=a_{c}-a_{v}$. Values for $a_{v}, a_{c}$, and a are listed in Table I.

The list of semiconductors in Table $I$ is divided into several blocks, each of which contains materials with similar characteristics. The model-solid approach is expected to give the most reliable results for lineups between materials belonging to the same block. When two semiconductors belong to different blocks, the resulting lineups should be regarded with more caution. We also point out that within the model-solid theory no distinction exists between different interface orientations. The band lineups at lattice-matched interfaces will therefore be independent of interface orientation, which has been confirmed by full interface calculations.' For strained-layer interfaces, the strains may of course depend on the particular orientation, and thus affect the lineups.

\section{RESULTS AND COMPARISON WITH EXPERIMENT}

Let us illustrate how to derive band offsets for a heterojunction $A / B$ starting from the values in Table I. For lattice-matched interfaces, the discontinuity in the average valence bands is

$$
\Delta \mathrm{E}_{\mathrm{v}, \mathrm{av}}=\mathrm{E}_{\mathrm{v}, \mathrm{av}}^{\mathrm{B}}-\mathrm{E}_{\mathrm{v}, \mathrm{av}}^{\mathrm{A}}
$$

The sign convention is such that $\Delta \mathrm{E}_{\mathrm{v}, \mathrm{av}}$ is positive when the valence band in $\mathrm{B}$ is higher in energy than the valence band in A. To obtain the position of the individual valence bands with respect to the average, the spin-orbit splitting $\Delta_{o}$ (also listed in Table I) has to be introduced. The topmost valence band in $\mathrm{A}$ is then given by

$$
\mathrm{E}_{\mathrm{v}}^{\mathrm{A}}=\mathrm{E}_{\mathrm{v}, \mathrm{a} v}^{\mathrm{A}}+\Delta_{\mathrm{o}}^{\mathrm{A}} / 3
$$

As an example, we find for $\mathrm{AlSb} / \mathrm{GaSb}: \Delta \mathrm{E}_{\mathrm{v}, \mathrm{av}}=(-6.25)-(-6.66)=0.41 \mathrm{eV}$, and $E_{\mathrm{v}}=0.41+(0.82 / 3)-(0.65 / 3)=0.47 \mathrm{eV}$. This value is very close to the experimental values: $0.41 \pm 0.1 \mathrm{eV}$, obtained by Gualtieri et al. from XPS measurements, ${ }^{8}$ and $0.45 \pm 0.08$ $\mathrm{eV}$, obtained by Menéndez et al. with a light-scattering technique. ${ }^{9}$

For strained-layer interfaces, one first has to determine the strain components in each of the materials. These depend on the boundary conditions at the pseudomorphic interface, the thickness of the layers, and macroscopic elastic constants, as described in 
Ref. 2. Herc we assume that the strain tensor is known for each material. The positions of $\mathrm{E}_{\mathrm{v}, \mathrm{av}}$ will be affected by the volume change in the layers. The relation

$$
\mathrm{E}_{\mathrm{v}, \mathrm{av}}=\mathrm{E}_{\mathrm{v}, \mathrm{av}}^{\mathrm{o}}+\mathrm{a}_{\mathrm{v}} \frac{\Delta \Omega}{\Omega}
$$

cxpresses $\mathrm{E}_{\mathrm{v}, \mathrm{av}}$ in terms of its value in the unstrained material (i.e. the equilibrium-volume value from Table I), the hydrostatic deformation potential for the valence band, $a_{v}$, and the fractional volume change $\Delta \Omega / \Omega=\left(\varepsilon_{x x}+\varepsilon_{y y}+\varepsilon_{z z}\right) . \Delta \mathrm{E}_{\mathrm{v}, \mathrm{av}}$ then follows immediately. The splitting of the valence band is now caused by uniaxial strain as well as by spin-orbit effects. The strain splitting can be obtained using the appropriate deformation potentials and strain components. Expressions for (111), (110), and (100) strains are listed in Ref. 2; the deformation potentials ( $b$ and d) can be calculated (see examples in Refs. 2,4), or taken from experiment. ${ }^{10}$ Finally, strain and spin-orbit splittings are combined ${ }^{2}$ to give the positions of the three valence bands with respect to $E_{v, a v}$, and consequently the valenceband offsets. Conduction bands can be positioned in a similar manner, using the $\mathrm{E}_{\mathrm{v}, \mathrm{av}}$ and band-gap values listed in Table $\mathrm{I}$, and including the appropriate shifts and splittings due to strain.

As an example for the derivation of lineups at a strained-layer interface, we choose a $\mathrm{ZnS} / \mathrm{ZnSe}$ interface. " A thin layer of pure $\mathrm{ZnS}$, deposited on a (001) ZnSe substrate, is subject to strains: $\varepsilon_{x x}=\varepsilon_{y y}=0.046, \varepsilon_{z z}=-0.058$. This results in a volume change $\Delta \Omega / \Omega=0.035$. From Table I and Eqn.(4):

$$
E_{\mathrm{v}, \mathrm{av}}^{\mathrm{ZnSc}}=-8.37 \mathrm{eV} \text {, and } E_{\mathrm{v}, \mathrm{av}}^{\mathrm{ZnS}}=-9.15+2.31 \times 0.035=-9.07 \mathrm{eV} \text {. }
$$

This leads to $\Delta \mathrm{E}_{\mathrm{v}, \mathrm{av}}=0.70 \mathrm{eV}$ (higher in $\mathrm{ZnSe}$ ). Adding in uniaxial strain and spin-orbit splittings ${ }^{11}$ leads to $\Delta \mathrm{E}_{\mathrm{v}}=0.58 \mathrm{eV}$. For the conduction bands, we find:

$$
\begin{aligned}
\mathrm{E}_{\mathrm{c}}^{\mathrm{ZnSe}} & =\mathrm{E}_{\mathrm{v}, \mathrm{av}}^{\mathrm{ZnSe}}+\Delta_{\mathrm{o}}^{\mathrm{ZnSe}} / 3+\mathrm{E}_{\mathrm{g}}^{\mathrm{ZnSe}}=-8.37+(0.43 / 3)+2.83=-5.40 \mathrm{eV}, \\
\mathrm{E}_{\mathrm{c}}^{\mathrm{ZnS}} & =\mathrm{E}_{\mathrm{v}, \mathrm{av}}^{\mathrm{ZnS}, \mathrm{o}}+\Delta_{\mathrm{o}}^{\mathrm{ZnS} / 3}+\mathrm{E}_{\mathrm{g}}^{\mathrm{ZnS}}+\mathrm{a}_{\mathrm{c}} \times \Delta \Omega / \Omega \\
& =-9.15+(0.07 / 3)+3.84+(-4.09) \times 0.035=-5.43 \mathrm{eV} .
\end{aligned}
$$

This results in $\Delta \mathrm{E}_{\mathrm{c}}=0.03 \mathrm{eV}$. This very small value for $\Delta \mathrm{E}_{\mathrm{c}}$ is characteristic for all $\mathrm{ZnS} / \mathrm{ZnSe}$ intcrfaces, and agrees with experimental observations. ${ }^{11}$

Good agreement between model-solid predictions and experiment was also found for $\mathrm{Si} / \mathrm{Ge}$ strained-layer interfaces, ${ }^{5}$ and for many lattice-matched junctions. ${ }^{1,4,5.12}$ We have also compared the lineups derived from Table I with those calculated from full selfconsistent interface calculations, where available. ${ }^{1,2,4,5,12}$ The results of the model are almost always within $0.2 \mathrm{eV}$ of the fully self-consistent values, both for lattice-matched and strained-layer interfaces. This theoretical justification, along with favorable comparisons with experiment, gives us confidence in the model and values presented here.

Table I only contains results for pure materials. Results for alloys can, to a reasonable approximation, be obtained by interpolation. ${ }^{2}$ As an example, we consider $\mathrm{Ga}_{47} \mathrm{In}_{53} \mathrm{As}$ grown on a (100) InP substrate and find $\Delta \mathrm{E}_{\mathrm{v}}=0.34 \mathrm{eV}$. Lang et al. ${ }^{13}$ applied the novel technique of admittance spectroscopy to this lattice-matched interface, leading to a valence-band offset of $0.35 \mathrm{eV}$; Skolnick et al. ${ }^{14}$ found $\Delta \mathrm{E}_{\mathrm{v}}=0.38 \mathrm{eV}$. Both values are in excellent agreement with our theoretical prediction. When the composition of the $\mathrm{Ga}_{x} \mathrm{In}_{1-x}$ As alloy is changed, strains will be introduced during pseudomorphic growth on a 
InP substrate. The band alignments in the resulting heterostructures have recently been analyzed by People, ${ }^{15}$ using our values for the lineups.

\section{CONCLUSIONS}

We have presented a theoretical model to calculate band lineups at lattice-matched and straincd-layer interfaces, and tabulated parameters to calculate band lineups for a wide variety of semiconductors. The important effects due to strains in the layers were emphasized. These strains are determined by the lattice constants (i.e. choice of materials and alloy composition), the boundary conditions (i.e. choice of substrate), and the thickness of the layers (in a free-standing superlattice). This provides wide flexibility in the design of new heterostructures. The model and values presented here provide a basis for analysis and design of novel interface structures.

\section{ACKNOWLEDGMENTS}

Thanks are due to R. M. Martin and J. Tersoff for helpful discussions.

\section{REFERENCES}

' C. G. Van de Walle and R. M. Martin, Phys. Rev. B 35, 8154 (1987).

${ }^{2}$ C. G. Van de Walle and R. M. Martin, Phys. Rev. B 34, 5621 (1986).

${ }^{3}$ See the discussion in Ref. 2.

${ }^{4}$ C. G. Van de Walle, Ph. D. Dissertation, Stanford University (1986).

${ }^{5}$ C. G. Van de Walle and R. M. Martin, J. Vac. Sci. Technol. B 4, 1055 (1986).

'G. B. Bachelet, D. R. Hamann and M. Schluter, Phys. Rev. B 26, 4199 (1982).

${ }^{7}$ Atomic configurations are listed in Ref. 2, and were obtained from tight-binding calculations by D. J. Chadi (private communication).

${ }^{*}$ G. P. Gualticri, R. G. Nuzzo, R. J. Malik, J. F. Walker, L. C. Feldman, W. A. Sunder, and G. P. Schwartz, J. Vac. Sci. Technol. B 5, 1284 (1987).

' J. Menéndez, A. Pinczuk, D. J. Werder, J.P. Valladares, T. H. Chiu, and W. T. Tsang, Solid State Commun. 61, 703 (1987).

${ }^{10}$ Landolt-Börnstein, Numerical Data and Functional Relationships in Science and Technology (Springer, New York, 1982), Group III, Vol. 17 a-b.

"K. Shahzad, D. J. Olego and C. G. Van de Walle (to be published).

${ }^{12}$ C. G. Van de Walle and R. M. Martin, J. Vac. Sci. Technol. B5, 1225 (1987).

${ }^{13}$ D. V. Lang, M. B. Panish, F. Capasso, J. Allam, R. A. Hamm, A. M. Sergent, and W. T. Tsang, Appl. Phys. Lett. 50, 736 (1987).

${ }_{14}$ M. S. Skolnick, P. R. Tapster, S. J. Bass, A. D. Pitt, A. Apsley, and S. P. Aldred, Semicond. Sci. Technol. 1, 29 (1986).

${ }^{15}$ R. People, J. Appl. Phys. 62, 2551 (1987). 